\title{
First theoretical determination of relative biological effectiveness of very high energy electrons
}

\author{
Authors: R. Delorme ${ }^{1,2,}{ }^{*}$, T. Masilela ${ }^{3}$, C. Etoh ${ }^{2}$, F. Smekens ${ }^{4}$, Y. Prezado ${ }^{3}$ \\ ${ }^{1}$ Univ. Grenoble Alpes, CNRS, Grenoble INP, LPSC-IN2P3, 38000 Grenoble, France \\ ${ }^{2}$ Imagerie et Modélisation en Neurobiologie et Cancérologie (IMNC), CNRS Univ Paris-Sud, Université \\ Paris-Saclay, F-91400, Orsay, France \\ ${ }^{3}$ Institut Curie, Orsay Research Centre, CNRS UMR3347, INSERM U1021, University Paris Saclay, France \\ ${ }^{4}$ Dosisoft, R\&D Medical Physics, Cachan, France \\ *Corresponding authors: rachel.delorme@lpsc.in2p3.fr
}

\begin{abstract}
Purpose: Very high energy electrons (VHEEs) present promising clinical advantages over conventional beams. They are able to target deep-seated tumors due to their increased range and improved penumbra compared to low energy electrons. VHEEs are also relatively insensitive to tissue heterogeneities, and are able to be electromagnetically scanned. These advantages facilitate their use in conjunction with spatial fractionation techniques or FLASH irradiations. However, the lack of radiobiological data concerning their biological efficacy is a limiting factor. This study aims to characterize different VHEE beams against clinically available beams by making use of Monte Carlo (MC) based numerical simulations to compare their macroand microdosimetric properties.
\end{abstract}

Methods: All simulations were performed on GATE version 8.2. A solid water phantom was irradiated by the following beams: 5, 20, 100, and $300 \mathrm{MeV}$ electrons, a ${ }^{60} \mathrm{Co}$ source (1.25 MeV photons), $105 \mathrm{MeV}$ protons, $194.2 \mathrm{MeV} /$ nucleon ${ }^{12} \mathrm{C}$ ions, and $262 \mathrm{MeV} /$ nucleon ${ }^{20} \mathrm{Ne}$ ions. The dose-averaged linear energy transfer $\left(\overline{L_{d}}\right)$ was evaluated as the macrodosimetric quantity of interest. On a microscopic scale, the lineal energy $y$ was used in order to account for the stochasticity of irradiations. The dose-mean lineal energy $\overline{y_{d}}$ and the lineal energy distribution described as a function of its dose density, $d(y)$, were calculated. A tissue equivalent proportional counter (TEPC) was implemented in GATE to record the lineal energy spectra. Finally, the modified microdosimetric kinetic model (MKM) was used to calculate the respective cell survival curves using biological parameters of HSG cell line and the lineal energy spectra as inputs.

Results: From the macrodosimetric point of view, VHEEs present a potential improved biological efficacy over clinical photon/electron beams due to their increased $\overline{L_{d}}$. At a depth of $4 \mathrm{~cm}$ in water, the ratio of $300 \mathrm{MeV} \overline{L_{d}}$ values to other particles is $0.2,1.9,3.2$, and 2.4 for protons, $100 \mathrm{MeV}$ electrons, $20 \mathrm{MeV}$ electrons, and photons respectively. The microdosimetric data, however, suggests no increased biological effectiveness of VHEEs over clinical electron beams. No significant differences were found between their lineal energy spectra nor their $\overline{y_{d}}$ depth profiles. Correspondingly, application of the MKM yielded similar cell survival curves, resulting in RBE values for VHEEs of approximately 1. Furthermore, RBE values of 1.2, 2.9 and 3.3 were obtained for proton, carbon-ion and neon-ion beams respectively. $\overline{L_{d}}$ values above 200 $\mathrm{keV} / \mu \mathrm{m}$ and lineal energies as high as $2000 \mathrm{keV} / \mu \mathrm{m}$ were obtained in the Bragg peak region for neon-ions.

Conclusions: This study represents a first step towards a full evaluation of the biological efficacy of VHEE beams. The biologically relevant information obtained from these theoretical MC simulations could be used to complement further experiments which explore the radiobiological response to VHEE treatment. 

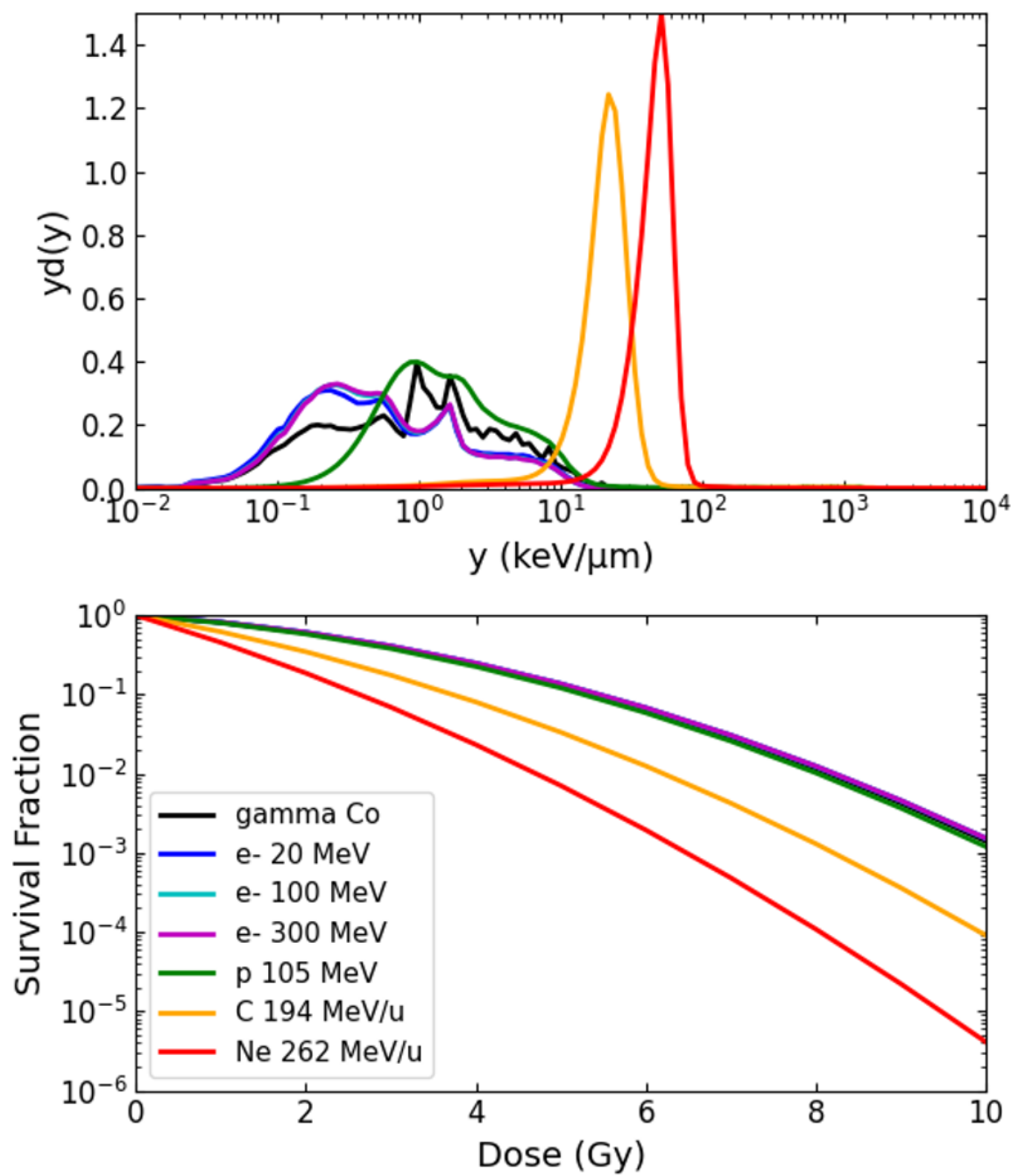

Figure 1: Comparison of microdosimetric spectra yd(y) for beams of photons, electrons, protons, carbon and neon ions (upper panel), and corresponding cell survival curves obtained with the MKM (lower panel), calculated at a depth of $4 \mathrm{~cm}$ in water (plateau region for ions).

Table 1: Calculated values of $\overline{y_{d}}$ and $R B E_{10}$ for $20 \mathrm{MeV}$ and $300 \mathrm{MeV}$ electron, proton, carbon and neon ion beams at 4 and $8.2 \mathrm{~cm}$ in depth.

\begin{tabular}{lcccc}
\hline \multicolumn{1}{c}{ Particle } & $\mathbf{y}_{\mathbf{d}}-\mathbf{4} \mathbf{c m}$ & $\mathbf{Y}_{\mathbf{d}}-\mathbf{8 . 2} \mathbf{c m}$ & $\mathrm{RBE}_{\mathbf{1 0}}-\mathbf{4} \mathbf{c m}$ & $\mathrm{RBE}_{\mathbf{1 0}}-\mathbf{8 . 2} \mathbf{~ c m}$ \\
\hline $20 \mathrm{MeV}$ electrons & 1.31 & 1.43 & 0.99 & 0.99 \\
$300 \mathrm{MeV}$ electrons & 1.25 & 1.29 & 0.99 & 0.99 \\
$105 \mathrm{MeV}$ protons & 5.34 & 12.86 & 1.02 & 1.24 \\
$194 \mathrm{MeV} / \mathrm{n}$ Carbon ions & 25.17 & 325.4 & 1.44 & 2.94 \\
$262 \mathrm{MeV} / \mathrm{n}$ Neon ions & 45.73 & 990.7 & 2.03 & 3.32 \\
\hline
\end{tabular}

Keywords: Very high-energy electrons, Radiation Therapy, microdosimetry, GATE, microdosimetric kinetic model 